\title{
A linear programming approach to fitting an upper quadratic boundary line to natural rubber data
}

\author{
B.M.S.G. Banneheka ${ }^{1 *}$, M.P. Dhanushika ${ }^{2}$, Wasana Wijesuriya ${ }^{3}$ and Keminda Herath ${ }^{4}$ \\ ${ }^{I}$ Department of Statistics and Computer Science, Faculty of Applied Sciences, University of Sri Jayewardenepura, Gangodawila, Nugegoda. \\ ${ }^{2}$ Department of Computational Mathematics, Faculty of Information Technology, University of Moratuwa, Katubedda, Moratuwa. \\ ${ }^{3}$ Biometry Section, Rubber Research Institute of Sri Lanka, Dartonfield, Agalawatta. \\ ${ }^{4}$ Department of Agribusiness Management, Faculty of Agriculture and Plantation Management, Wayamba University of Sri Lanka, Makandura.
}

Revised: 22 November 2012; Accepted: 20 December 2012

\begin{abstract}
In the context of biology, the problem of finding the maximum or minimum response in a cause and effect relationship is recognized as boundary line fitting. Frequently used methods involve fitting curves through the boundary points using the least square principle. However, identification of boundary points especially when there are not any multiple responses at values of the explanatory variable is ad hoc. Existing methods involve dividing the range of explanatory variable into bins and considering points with the maximum response or the response above some value in each bin. However, the results depend heavily on the way of dividing into bins and the number of bins. There is no agreement on the best way of dividing or the number of bins. Furthermore, the least square line is not consistent with the theory of limiting response because it goes through the points rather than going above all the points or below all the points, representing the boundary. This paper presents a new method that avoids all the above drawbacks. It involves the theory of linear programming. The proposed method has been compared with commonly used methods by using simulated data and shown to perform better. The method is illustrated by applying to experimental data on the response of latex yield of natural rubber to leaf nutrient concentrations.
\end{abstract}

Keywords: Biological data, bootstrap confidence intervals, limiting response, linear programming.

\section{INTRODUCTION}

Biological data are often generated as a consequence of a biological response of a dependent variable against one or more covariates, which explain the variability of the response in a biological system or a process. This can be further interpreted as a set of data generated as a result of a cause and effect relationship in a biological process.
In this study, the leaf nutrient concentrations of the major elements, $\mathrm{N}, \mathrm{P}, \mathrm{K}$ and $\mathrm{Mg}$ used in the determination of fertilizer recommendations for rubber was considered. The critical levels for these elements are decided by their response to the latex yield of rubber. These kind of relationships need to be built in a reliable way for the routing exercise of fertilizer recommendations to the rubber sector.

The cause and effect relationships in a biological process are of simple to very complex in nature and of greater interest to biologists and researchers in understanding behavioural and functional features of a process. A variety of statistical models are used to figure out the cause and effect relationships found in various biological processes. Most of the underlying statistical models; viz. linear regression, non linear regression and smoothing techniques, basically explain the average response of a variable against a covariate or a set of covariates. Even if it is statistically sound for prediction purposes, it is not biologically meaningful unless the dependent variable can be treated as an additive combination of all explanatory variables in a biological context (Milne et al., 2006).

The maximum, minimum or limiting response under given conditions are generally important in the context of biology. Webb (1972) suggested that the upper boundary or the lower boundary of a plot that indicates the biological response may be of greater biological interest than the line of the best fit through the set of data. Further, he proposed the boundary line as a conceptual model for the interpretation of a plot of two biological 
variables where the dependent variable is some biological response to some set of independent variables, one of which is represented on the abscissa of the plot (Milne et al., 2006). The upper or lower edges of a set of data in a plot are considered as the upper boundary line or the lower boundary line, respectively. The upper boundary comprised the maximum responses of the dependent variable at particular levels of a covariate, while the lower boundary includes the set of minimum observations of the dependent variable at respective levels of the covariate.

Biologists have found that boundary line model is persuasive and of practical interest in various contexts. Casanova et al. (1999); Schmidt et al. (2000); Shatar and McBratney (2004); Gunarathna et al. (2004); Lewandowski and Schmidt (2006) and Feiziasl et al. (2010) have applied boundary line method to assess the cause-effect relationships in biological data in crop growing environments. The boundary line may also represent the limiting response to available water (independent variable) in a multiplicative limiting response model, which has been used to trace gas emissions (dependent variable) from soil (Rolston et al., 1984; Milne et al., 2005; Pringle \& Lark, 2006). The boundary line approach has also been used for modelling within field yield variation as a basis for recommendations on site-specific management (Shatar \& McBratney, 2004) and in specifying nutrient standards of durian (Poovarodom \& Chatuepot, 2002). However, in most of the applications, methods of estimating the boundary line are ambiguous and lacking an underpinning statistical model. Further, Milne et al. (2006) have highlighted that the algorithms proposed by Webb (1972); Schnug et al. (1996); Kitchen et al. (1999); Tang et al. (1999); Schmidt et al. (2000) and Shatar and McBratney (2004) have not been widely adopted due to their impromptu nature.

In general, the existing methods to fit the boundary lines are based on the least squares regression. Detection of outliers, identification of plausible points on the boundary and curve fitting are three major steps that are involved in the least squares method of fitting boundary line. The measurement errors are expected to be removed during the outlier detection. Various methods to identify outliers before fitting a boundary line to a dataset can be found in literature. Calculating Mahalanobis distance is a popular method in the literature to remove outliers. Shatar and McBratney (2004) have removed data points with the largest $5 \%$ of Mahalanobis distances before fitting of the boundary line. Some researchers count the near neighbours of each observation and remove the observations, which have a fewer neighbours than some threshold. A drawback of these methods is the possible removal of a data point that may not actually be an outlier. Therefore, a useful data point also can be wrongly deleted as an outlier (Milne et al., 2006). Simple graphical methods like scatter plots and box plots can also be used to identify outliers. However, information obtained from these graphs can be subjective and therefore, understanding of the dataset is essential to discard the outliers of the practical dataset.

In the process of identifying points that fall on the boundary, data are grouped into subdivisions (bins) with respect to the independent variable (x-axis). However, the number of subdivisions, sizes of subdivisions and underlying method of grouping have not been clearly defined yet. As Shatar and McBratney (2004) have pointed out, the method chosen to partition the data into bins affects the results. If the bin width is too narrow, the set of bin maxima will be noisy, and the fitted model will tend towards simple regression. With too wide bin sizes, the form of the boundary response will be poorly represented. Location of bins also affects the results.

Three commonly used methods to fit the boundary line are briefly mentioned below. For full details the reader may refer to Kitchen et al. (1999); Schmidt et al. (2000); and Shatar and McBratney (2004). All three methods involve dividing the range of $\mathrm{x}$ values into bins and least square estimation. The recommended number of bins in the literature was 8 to 10 . However, the robustness of these methods on the fitted line has not yet been studied. The methods are described assuming that the $\mathrm{x}$ values have been divided into 10 bins.

1. Find the maximum y within each bin. Let them be denoted by $\mathrm{v}_{1}, \mathrm{v}_{2}, \ldots, \mathrm{v}_{10}$. Let $\mathrm{u}_{1}, \mathrm{u}_{2}, \ldots, \mathrm{u}_{10}$ be the $\mathrm{x}$ values corresponding to those $\mathrm{v}_{1}, \mathrm{v}_{2}, \ldots, \mathrm{v}_{10}$, respectively. Fit the least square boundary line using the points $\left(\mathrm{u}_{1}, \mathrm{v}_{1}\right),\left(\mathrm{u}_{2}, \mathrm{v}_{2}\right), \ldots,\left(\mathrm{u}_{10}, \mathrm{v}_{10}\right)$. We call this method 'LS1'.

2. Let $\mathrm{u}_{1}, \mathrm{u}_{2}, \ldots, \mathrm{u}_{10}$ be the middle $\mathrm{x}$ values of the 10 bins. Find the 99th percentile of the y values within each bin. Let them be denoted by $v_{1}, v_{2}, \ldots, v_{10}$. Fit the least square boundary line using the points $\left(\mathrm{u} 1, \mathrm{v}_{1}\right),\left(\mathrm{u}_{2}, \mathrm{v}_{2}\right), \ldots,\left(\mathrm{u}_{10}, \mathrm{v}_{10}\right)$. We call this method 'LS2'.

3. Find the $95^{\text {th }}$ percentile of the $y$ values within each bin. For each bin, consider the points for which $y$ values are equal to or above the $95^{\text {th }}$ percentile. Fit the least squares boundary line using all such points in the 10 bins. We call this method 'LS3'.

We compare our method with the above three methods. 


\section{METHODS AND MATERIALS}

Even though a considerable amount of literature is available on the boundary line fitting, none of them have a concrete mathematical definition for the boundary line. Therefore, we start by offering a definition for an upper boundary line. Suppose $\mathrm{X}$ is some explanatory (independent) variable, $\mathrm{Y}$ is a dependent variable and $\mathrm{X}$ and $\mathrm{Y}$ are jointly distributed with some density function $f_{X, Y}(x, y)$ on the domain $D_{X, Y}$. Further, suppose that there exists some function $\mathrm{g}(\mathrm{x} ; \beta)$ in the domain $\mathrm{D}_{\mathrm{x}}$ such that

$\operatorname{Pr}(\mathrm{Y} \leq \mathrm{g}(\mathrm{x} ; \beta) \mid \mathrm{X}=\mathrm{x})=1 \forall \mathrm{x} \in \mathrm{Dx}$

and for any $\varepsilon>0$,

$$
\operatorname{Pr}(\mathrm{Y} \leq \mathrm{g}(\mathrm{x} ; \beta)-\varepsilon \mid \mathrm{X}=\mathrm{x})<1 \forall \mathrm{x} \in \mathrm{Dx}
$$

Then, $\mathrm{g}(\mathrm{x} ; \beta)$ can be considered as an upper boundary line. The condition (1) simply states the requirement that all the values of the response variable for a given value of $\mathrm{X}$ must be below the upper boundary line. The condition (2) eliminates all the other curves that are above the real boundary line, still satisfying (1). Here, $\beta$ is a vector of unknown coefficients. For example, for the straight line boundary $\mathrm{g}(\mathrm{x} ; \beta)=\beta_{0}+\beta_{1} \mathrm{x}$ with $\beta=\left(\beta_{0}, \beta_{1}\right)^{\mathrm{T}}$, and for the quadratic boundary function $\mathrm{g}(\mathrm{x} ; \beta)=\beta_{0}+\beta_{1} \mathrm{x}+\beta_{2} \mathrm{x}^{2}$, with $\beta=\left(\beta_{0}, \beta_{1}, \beta_{2}\right)^{\mathrm{T}}$. The boundary line $\mathrm{g}(\mathrm{x} ; \beta)$ can also be any other suitable function like a polynomial of order 3 or more or a non linear function.

A lower boundary line can be defined analogously. The discussion in this paper is restricted to estimating a quadratic upper boundary line. However, the methods proposed here can be easily adapted for an upper or lower polynomial boundary line of order 3 or more.

\section{Fitting the boundary line}

The method of least square and the method of maximum likelihood are the most commonly used methods for fitting curves. The method of least square is suitable when the line of interest goes through the data so that roughly equal number of observations is above and below the line. A dataset consistent with requirements (1) and (2) should contain all the points below the actual boundary line. Therefore, the least square method is not suitable for estimating a boundary line defined as above. The method of maximum likelihood can be used only if a parametric model is available for the joint density $f_{X, Y}$. Finding a suitable model for $f_{X, Y}$ is usually difficult and estimation of the boundary line using the method of maximum likelihood can be more difficult due to additional parameters in $\mathrm{f}_{X, Y}$. A much simpler solution is presented in this paper.

Suppose there exists a boundary line of the form $\mathrm{g}(\mathrm{x} ; \beta)=\beta_{0}+\beta_{1} \mathrm{x}+\beta_{2} \mathrm{x}^{2}$ and we have a set of $\mathrm{n}$ independent pairs of observations $\left(\mathrm{x}_{1}, \mathrm{y}_{1}\right),\left(\mathrm{x}_{2}, \mathrm{y}_{2}\right), \ldots$, $\left(x_{n}, y_{n}\right)$. Further, suppose there are $m(m \leq n)$ different $x$ values among $\mathrm{x}_{1}, \mathrm{x}_{2}, \ldots, \mathrm{x}_{\mathrm{n}}$ and let them be denoted by $\mathrm{u}_{1}, \mathrm{u}_{2}, \ldots \mathrm{u}_{\mathrm{m}}$. Let $\mathrm{v}_{\mathrm{i}}$ be the maximum of $\mathrm{y}$ values when $\mathrm{X}$ $=u_{i}$. We use the subset $\left(u_{1}, v_{1}\right),\left(u_{2}, v_{2}\right), \ldots,\left(u_{m}, v_{m}\right)$ for our method. This is not a waste of information, because given these points, all the other points do not contain any further information about the upper boundary. This is because we know that the upper boundary must go above these points and it is enough for us that the boundary line goes as close as possible to these points.

We suggest to use $\hat{\beta}_{0}, \hat{\beta}_{1}$ and $\hat{\beta}_{2}$ that minimize

$$
\sum_{i=1}^{m}\left(\hat{\beta}_{0}+\hat{\beta}_{1} \mathrm{u}_{\mathrm{i}}+\hat{\beta}_{2} \mathrm{u}_{\mathrm{i}}^{2}-\mathrm{v}_{\mathrm{i}}\right)
$$

subject to

$$
\hat{\beta}_{0}+\hat{\beta}_{1} u_{i}+\hat{\beta}_{2} u_{i}^{2} \geq v_{i}, i=1,2, \ldots, m
$$

as the estimates of $\hat{\beta}_{0}, \hat{\beta}_{1}$ and $\hat{\beta}_{2}$, respectively. These two equations force the fitted line to go above all the data points while being as close as possible to the maximum points.

Note that $\sum_{i=1}^{m} \mathrm{v}_{\mathrm{i}}$ in (3) can be ignored without changing the solution, since it is a constant for a given dataset. Letting

$$
\mathrm{a}_{0}=\mathrm{m}, \mathrm{a}_{1}=\sum_{\mathrm{i}=1}^{\mathrm{m}} \mathrm{u}_{\mathrm{i}} \text {, and } \mathrm{a}_{2}=\sum_{\mathrm{i}=1}^{\mathrm{m}} \mathrm{u}_{\mathrm{i}}^{2}
$$

it is easy to see that the above is a simple linear programming problem to determine $\hat{\beta}_{0}, \hat{\beta}_{1}$ and $\hat{\beta}_{2}$ so that

$$
\mathrm{Q}=\mathrm{a}_{0} \hat{\beta}_{0}+\mathrm{a}_{1} \hat{\beta}_{1}+\mathrm{a}_{2} \hat{\beta}_{2}
$$

is minimized subject to constraints

$$
\hat{\beta}_{0}+u_{i} \hat{\beta}_{1}+u_{i}^{2} \hat{\beta}_{2} \geq v_{i}, i=1,2, \ldots, m
$$

This linear programming problem can be solved very easily with the help of any software that support linear programming. We used the package 2.11 .1 (2010) to calculate $\hat{\beta}_{0}, \hat{\beta}_{1}$ and $\hat{\beta}_{2}$. We call this method 'LP'. 


\section{Illustrations of methods}

This study employs both simulated and experimental data to describe the appropriateness of the proposed methods. The experimental data were collected from the survey programme of site specific fertilizer recommendation based on soil and foliar nutrients conducted by the Soils and Plant Nutrition Department of the Rubber Research Institute of Sri Lanka (RRISL). Leaf nitrogen and potassium concentrations (\%) together with latex yields (kg/ha/year) of different fields surveyed in 2009 were used in the study.

In this study, as an initial step, we identified and removed outliers by simply using graphical methods. Graphical representations of real data obtained from the Soils and Plant Nutrition Department of RRISL are shown in Figure 1. Box plot of yield data, which is shown in Figure 1 (a) was simply used to identify the outliers. When detecting outliers it is not enough to rely only on the information given by the box plot. Therefore a scatter plot, which shows the relationship between latex yields (kg/ha) and leaf nitrogen concentration (\%) was drawn as shown in Figure 1 (b). Two unusual observations, which have been circled could be seen in the scatter plot but only one point was marked as an outlier in the box plot. In this situation, knowledge of the field situation was necessary to take a decision. Therefore under the guidance of experts in the rubber field, the situation was carefully considered and the decision that both points were outliers was taken.
There are 117 observations in this dataset after removing outliers. Figure 2 illustrates the above four methods. The variables $\mathrm{X}$ and $\mathrm{Y}$ of the dataset are the leaf nitrogen concentration (\%) and the yield of latex $(\mathrm{kg} / \mathrm{ha})$, respectively. According to the scatter plot of the data, it appears reasonable to assume that there exists a quadratic upper boundary line. The bins used in 'LS1', 'LS2', and 'LS3' are shown by the dotted vertical lines. In 'LS1', 'LS3' and 'LP', the points used to fit the boundary line are marked with circles. These are subsets of the original sample. In 'LS2' the points used to fit the boundary are marked with ' $x$ '. This is a new set of points since $x$ values are the mid-points of the bins. It is clear that the 'LP' method uses more observations than the other methods to fit the boundary line. Hence, this method assures preservation of more information in the original sample compared to the other methods.

Since the actual boundary line is unknown for the real dataset, it is not possible to illustrate the overall closeness of the fitted boundary lines to the actual boundary line. Therefore, we simulated data with a known upper boundary line. Figure 3 shows the true (known) upper boundary line and the fitted upper boundary lines for a simulated dataset from a known bivariate normal distribution with a known boundary line. The data have been generated to be similar to the actual dataset used in Figure 2. Figure 3 clearly shows that the line fitted using the 'LP' method is the closest to the true boundary line. This finding further confirms the applicability of the 'LP' method for fitting the boundary line.

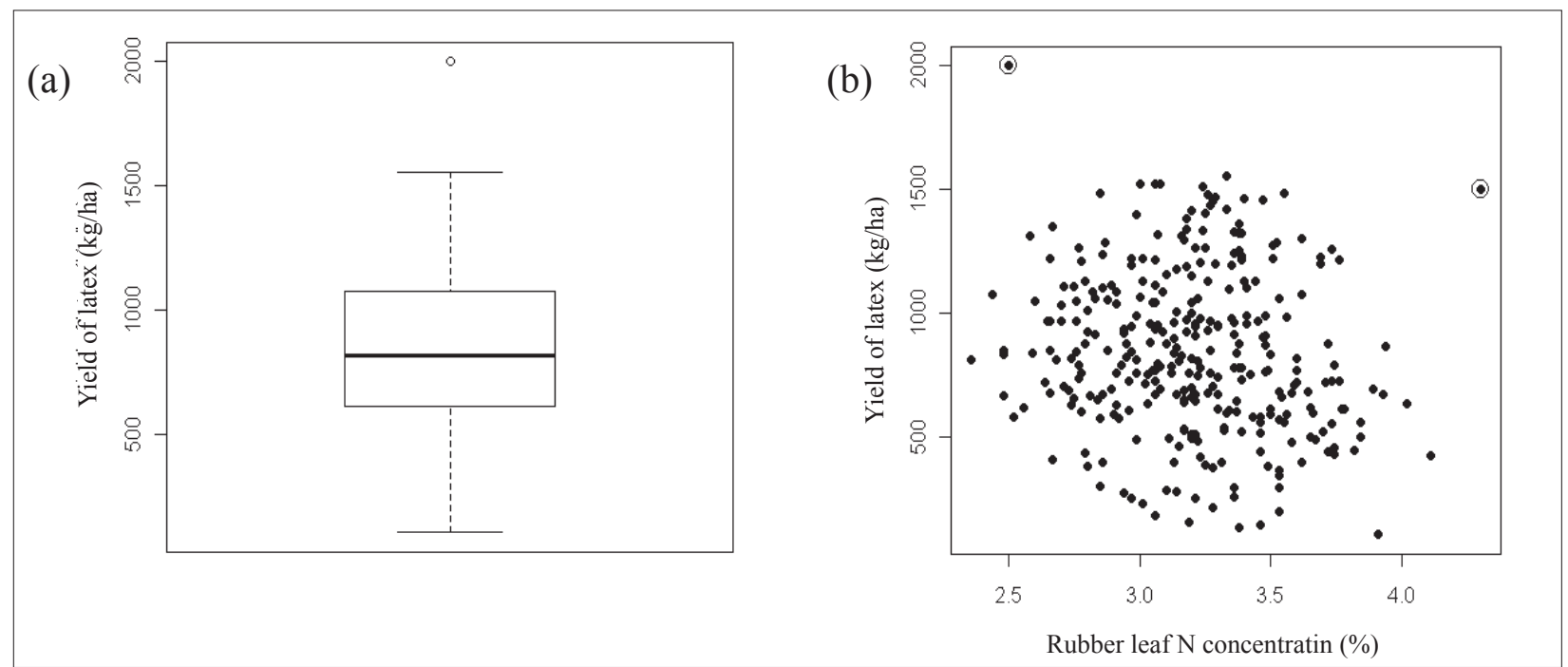

Figure 1: (a) Box plot of latex yield (kg/ha) and (b) scatter plot of latex yield (kg/ha) vs $\mathrm{N}$ concentration (\%). The points circled in (b) are the identified outliers. 


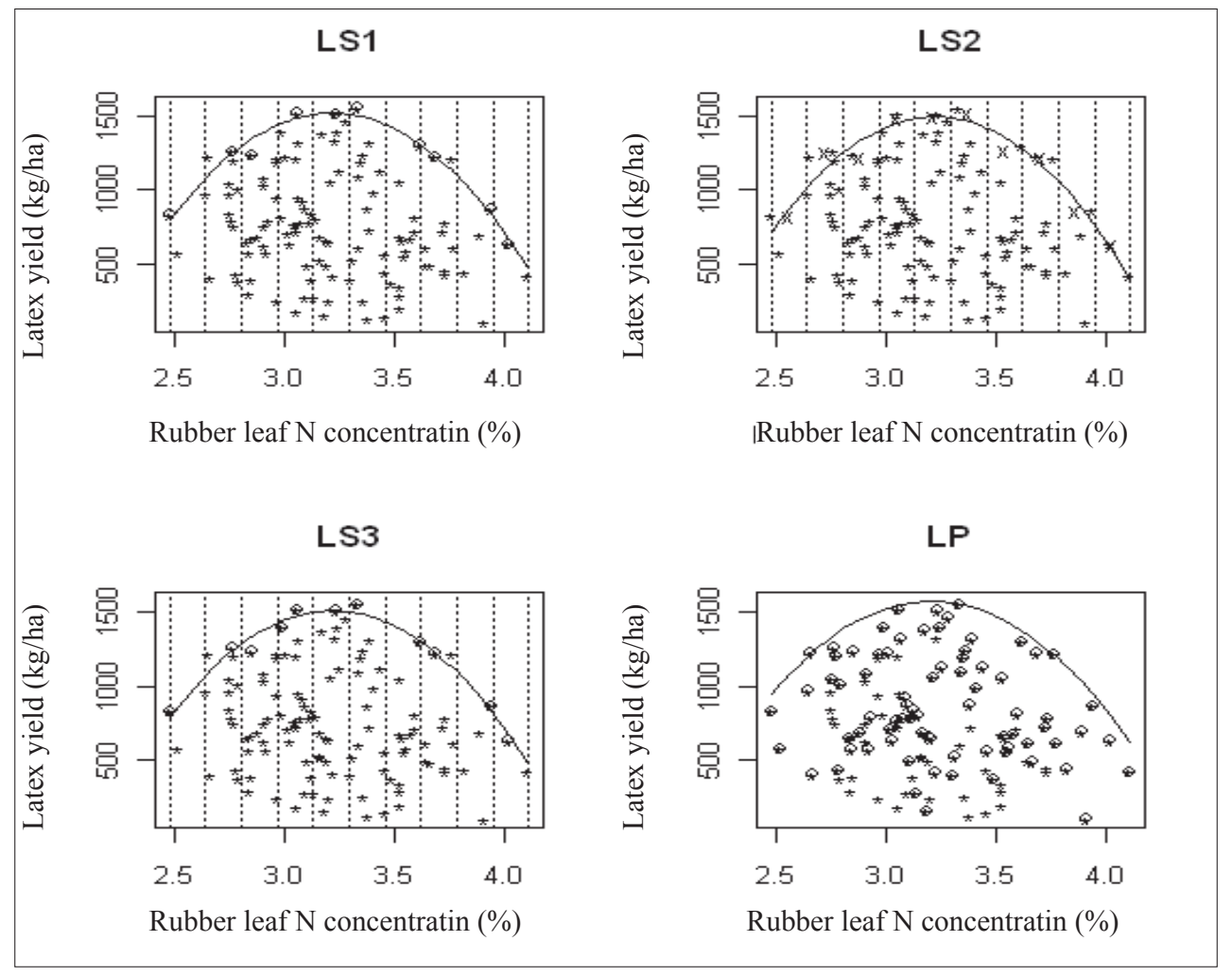

Figure 2: Fitted boundary lines for data on $\mathrm{N}$ concentration (\%) and latex yield ( $\mathrm{kg} / \mathrm{ha})$ using four methods. Vertical lines are the bines. The points circled in LS1, LS3 and LP and mid points indicated by X in LS2 are used for fitting the boundary.

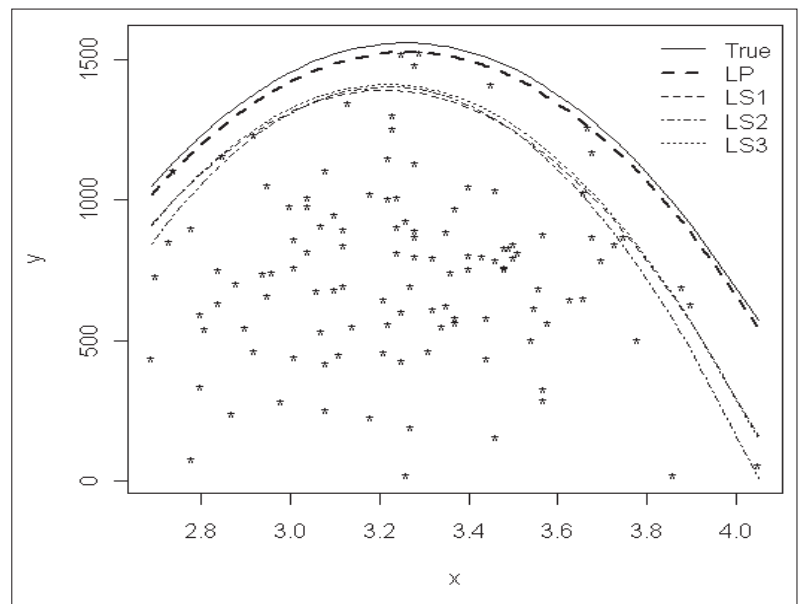

Figure 3: Fitted boundary lines and the true boundary line for a simulated data set

\section{Evaluation of method}

We compared our method 'LP' with the 3 methods 'LS1', 'LS2' and 'LS3' described above, using data generated from two known truncated bivariate normal distributions. In the first simulation study, the bivariate normal distribution and boundary line were selected so that the simulated data are similar to our real data on rubber leaf nitrogen concentration (X) and yield of latex (Y). In the second simulation study, they were selected so that the simulated data are similar to a real dataset on rubber leaf potassium concentration $(\mathrm{X})$ and yield of latex (Y). Sample sizes were 117 and 106, which were the same as our actual datasets. Parameters of the bivariate normal distribution and the boundary line used to generate data are given in Table 1. 
Table 1: Parameters of the bivariate normal distributions and respective boundary lines used in simulation studies

\begin{tabular}{ccc}
\hline Parameters & Simulation study 1 & Simulation study 2 \\
\hline Bivariate normal distribution & & \\
$\mu_{\mathrm{x}}$ & 3.22 & 0.90 \\
$\mu_{\mathrm{y}}$ & 767.68 & 821.40 \\
$\sigma_{\mathrm{x}}^{2}$ & 0.12 & 0.02 \\
$\sigma_{\mathrm{y}}^{2}$ & 128838.6 & 146851 \\
$\rho_{\mathrm{xy}}$ & -0.17 & 0.24 \\
$\mathrm{n}$ & 117 & 106 \\
Boundary line & & -4979 \\
$\beta_{0}$ & -15163 & 138879 \\
$\beta_{1}$ & 10264 & 7262 \\
$\beta_{2}$ & -1575 &
\end{tabular}

For a fixed known bivariate normal distribution with a known boundary line, the procedure was as follows:

1. create a sequence of 100 equally spaced $x$ values spanning the domain $\mathrm{D}_{\mathrm{x}}$ and name them $\mathrm{w}_{1}, \mathrm{w}_{2}, \ldots$, $\mathrm{W}_{100}$.

2. generate a dataset from the known truncated bivariate normal distribution with a known quadratic boundary line.

3. estimate the boundary line using each of LS1, LS2, LS3 and LP methods.

4. calculate

$G=\sqrt{\frac{1}{100} \sum_{i=1}^{100}\left[\widehat{\beta}_{0}+\widehat{\beta}_{1} w_{i}+\widehat{\beta}_{2} w_{i}^{2}-\left(\beta_{0}+\beta_{1} w_{i}+\beta_{2} w_{i}^{2}\right)\right]^{2}}$

as a measure of goodness of fit, under each method. $\mathrm{G}$ is a measure of overall closeness of the fitted boundary line to the actual boundary line. Note that even though the data are different from sample

Table 2: Values of $\bar{G}$ from two simulation studies

\begin{tabular}{ccc}
\hline Method & Simulation study 1 & Simulation study 2 \\
\hline LS1 & 192.96 & 237.27 \\
LS2 & 199.08 & 252.29 \\
LS3 & 198.76 & 238.83 \\
LP & 96.96 & 121.58 \\
\hline
\end{tabular}

Note: Each simulation study is based on 1000 simulations to sample, $\mathrm{w}_{1}, \mathrm{w}_{2}, \ldots, \mathrm{w}_{100}$ are fixed throughout the procedure.

5. repeat steps 2 to 4,1000 times.

6. calculate the mean $(\bar{G})$ of the 1000 values of $G$, for each method.

\section{RESULTS}

The values of $\bar{G}$ obtained from the simulation studies described in Table 1 are given in Table 2.

The fitted boundary line using the 'LP' method for the real data on leaf nitrogen concentration (\%) and the yield of latex $(\mathrm{kg} / \mathrm{ha})$ is:

$\hat{\theta} x)=-10482.67+7515.772 x-1171.247 \times 2$.

The fitted values for the boundary line at $\mathrm{x}=2.75,3.0$, 3.25 and 3.75 are given in Table 3. These results are shown in Figure 4. Based on the results in Figure 4, the maximum yields are obtained at 3.25 leaf nitrogen concentration (\%), when other factors are not limiting.

Table 3: Fitted boundary values by LP method at different $\mathrm{x}$ values

\begin{tabular}{cc}
\hline $\begin{array}{c}\mathrm{x} \text { (leaf nitrogen } \\
\text { concentration)(\%) }\end{array}$ & $\begin{array}{c}\text { Fitted boundary value }(\mathrm{x}), \\
\text { latex yield }(\mathrm{kg} / \mathrm{ha})\end{array}$ \\
\hline & \\
2.75 & 1328.1 \\
3.00 & 1523.4 \\
3.25 & 1572.3 \\
3.50 & 1474.7 \\
\hline
\end{tabular}




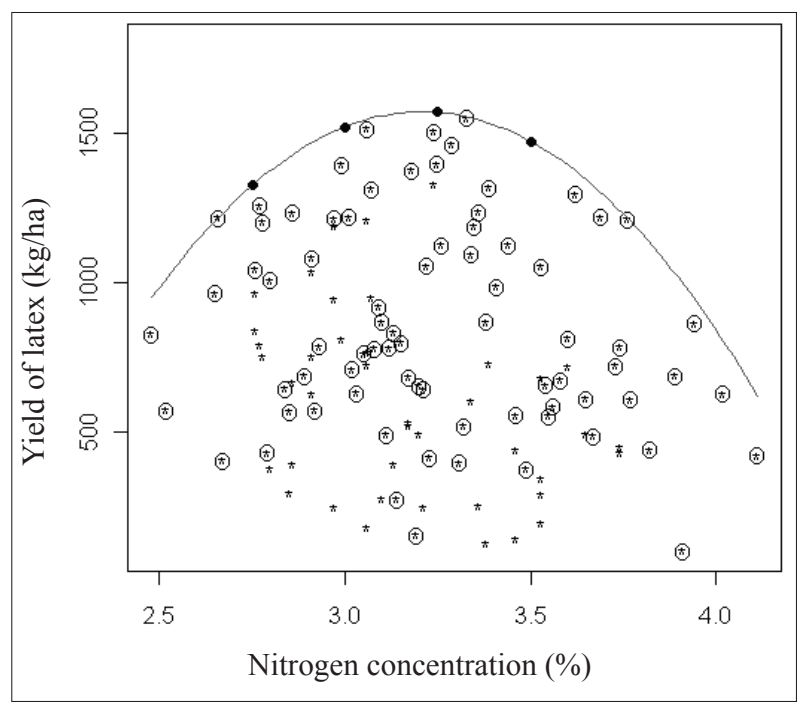

Figure 4: Fitted boundary line by LP method for data on $\mathrm{X}=$ nitrogen concentration $(\%)$ and $\mathrm{Y}=$ yield of latex $(\mathrm{kg} / \mathrm{ha})$. The points used for fitting the boundary line are circled. The fitted values for the boundary line at $x=2.75,3.0,3.25$ and 3.75 are shown by solid circles.

\section{DISCUSSION AND CONCLUSION}

According to Table 2, in both comparisons, the LP method has the minimum $\bar{G}$. We have carried out a large number of similar studies (not reported here) and in all those studies, the LP method had the minimum $\bar{G}$. This implies that out of the 4 methods compared, the LP method provides the best overall fit of the boundary line. Further, as pointed out earlier, it does not need to divide the range of $\mathrm{X}$ values into bins and employs more information from the dataset when compared to the other methods (Figure 2). The fitted boundary line is always on or above the data points of the sample, which is the strength of this method compared to the others.

This application also can be extended to leaf nutrient concentrations of other elements $(\mathrm{P}, \mathrm{K}$ and $\mathrm{Mg}$ ) to formulate a cost effective, technically efficient fertilizer elements to rubber plantations. Furthermore, this method can be adapted to fit boundary lines of forms other than quadratic functions.

\section{Future work}

In addition to a point estimate for the boundary value at a given value of $\mathrm{x}$, one would also be interested in mean squared error of the estimate or confidence interval. We investigated the possibility of using bootstrap methods for this purpose. Even though we were able to adjust the 'basic bootstrap method' to give coverage probability close the nominal confidence levels, the lengths of the intervals were too large for practical use. According to Bontempi (n.d.), the 'smoothness condition' required for the bootstrap methods to work is not true for extreme order statistics like the minimum and the maximum values. This may be the reason for failure of bootstrap methods in this problem. As such, the problem of finding confidence intervals is open for research.

\section{Acknowledgement}

We gratefully acknowledge Dr(Mrs.) Lalani Samarappuli, Head, Soils and Plant Nutrition Department, Rubber Research Institute of Sri Lanka for making the datasets available and for providing necessary information to carry out this research. We also thank the two anonymous referees for their valuable comments.

\section{REFERENCES}

1. Bontempi G. (n.d.). Resampling techniques for statistical modelling. Available at http://www.ulb.ac.be/di/map/ gbontelecares/boot1.pdf, Accessed 08 November 2012.

2. Casanova D.J., Goudriaan J.B. \& Epema G.F. (1999). Yield gap analysis in relation to soil properties in direct-seeded flooded rice. Geoderma 91: 191 - 216.

3. Feiziasl V., Jafarzadeh J., Amrl A., Ansarl Y., Mousavi S.B. \& Chenar M.A. (2010). Analysis of yield stability of wheat genotypes using new crop properties balance index (CPBI) method. Notulae Botanicae Horti Agrobotanici Cluj-Napoca 38(01): $228-233$.

4. Gunaratne G.P., Hettiarachchi L.S. \& Jayakody A.N. (2004). Boundary-line approach in specifying nutrient diagnosis ranges for vegetatively propagated tea in Sri Lanka. Proceedings of the First Symposium on Plantation Crop Research - Current Trends and Future Challenges, pp. $75-86$.

5. Kitchen N.R., Sudduth K.A. \& Drummond S.T. (1999). Soil electrical conductivity as a crop productivity measure for claypan soils. Journal of Production Agriculture 12: $607-617$.

6. Lewandowski I. \& Schmidt U. (2006). Nitrogen, energy and land use efficiencies of miscanthus, reed canary grass and triticale as determined by the boundary line approach. Agriculture, Ecosystems and Environment 112(04): $335-346$.

7. Milne A.E., Lark R.M., Addiscott T.M., Goulding K.W.T., Webster C.P. \& O'Flaherty S. (2005). Wavelet analysis of the scale- and location-dependent correlation of modelled and measured nitrous oxide emissions from soil. European Journal of Soil Science 56: 3 - 18. 
8. Milne A.E., Wheeler H.C. \& Lark R.M. (2006). On testing biological dataset for the presence of a boundary. Annals of Applied Biology 149: 213 - 222.

9. Poovardom S. \& Chatupote W. (2002). Boundary line approach in specifying durian nutrient standards, Poster Presentation, Paper no. 2319, $17^{\text {th }}$ World Congress of Soil Science, Bangkok, Thailand, $14-21$ August, pp. $1-6$.

10. Pringle M.J. \& Lark R.M. (2006). Spatial analysis of model error, illustrated by soil carbon dioxide emissions. Vadose Zone Journal 5: 168 - 183.

11. R Development Core Team (2010). R: A Language and Environment for Statistical Computing. R Foundation for Statistical Computing, Vienna, Austria. http://www. Rproject. org.

12. Rolston D.E., Rao P.S.C., Davidson J.M. \& Jessup R.E. (1984). Simulation of denitrification losses of nitrate fertilizer applied to uncropped, cropped, and manure amended field plots. Soil Science 137: 270 - 279.

13. Schmidt U., Thoni H. \& Kaupenjohann M. (2000). Using a boundary line approach to analyze N2O flux data from agricultural soils. Nutrient Cycling in Agro-ecosystems 57: $119-129$

14. Schnug E., Heym J. \& Achwan F. (1996). Establishing critical values for soil and plant analysis by means of the Boundary Line Development System (Bolides). Communications in Soil Science and Plant Analysis 27: $2739-2748$.

15. Shatar T.M. \& McBratney A.B. (2004). Boundary-line analysis of field-scale yield response to soil properties. The Journal of Agricultural Science 142: 553 - 560.

16. Tang Z., Chambers J.L. \& Barnett J.P. (1999). Characterization of optimum physiological responses of field-grown loblolly pine, $10^{\text {th }}$ Biennial Southern Silviculture Research Conference, Shreveport, Louisiana, 16 February, pp. $409-415$.

17. Webb R.A. (1972). Use of the boundary line in analysis of biological data. Journal of Horticultural Science 47: $309-319$. 